\title{
Quantitative Measurements of Oxidative Stress in Mouse Skin Induced by X-Ray Irradiation
}

\author{
Cuiping Chi, ${ }^{a, b}$ Ryoko Tanaka, ${ }^{a, c}$ Yohei OKudA, ${ }^{a, d}$ Nobuo Ikota, ${ }^{a}$ Haruhiko Yamamoto, ${ }^{c}$ \\ Shiro Urano, ${ }^{d}$ Toshihiko Ozawa, ${ }^{a, b}$ and Kazunori ANZAI ${ }^{*, a}$ \\ ${ }^{a}$ Redox Regulation Research Group, National Institute of Radiological Sciences; 4-9-1 Anagawa, Inage-ku, Chiba \\ 263-8555, Japan: ${ }^{b}$ Graduate School of Science and Technology, Chiba University; 1-33 Yayoi-cho, Inage-ku, Chiba \\ 260-8675, Japan: ${ }^{~}$ Department of Applied Bioscience, Faculty of Science, Kanagawa University; Hiratsuka 259-1293, \\ Japan: and ${ }^{d}$ Department of Applied Chemistry, Faculty of Engineering, Shibaura Institute of Technology; Tokyo 108-0014, \\ Japan. Received June 13, 2005; accepted August 26, 2005
}

To find efficient methods to evaluate oxidative stress in mouse skin caused by X-ray irradiation, several markers and methodologies were examined. Hairless mice were irradiated with $50 \mathrm{~Gy}$ X-rays and skin homogenates or skin strips were prepared. Lipid peroxidation was measured using the skin homogenate as the level of thiobarbituric acid reactive substances. The level of lipid peroxidation increased with time after irradiation and was twice that of the control at $78 \mathrm{~h}$. ESR spectra of skin strips showed a clear signal for the ascorbyl radical, which increased with time after irradiation in a manner similar to that of lipid peroxidation. To measure levels of glutathione (GSH) and its oxidized forms (GSSG) simultaneously, two HPLC methods, sample derivatization with 1-fluoro-2,4-dinitrobenzene and detection with a $U V$ detector (method $A$ ) and no derivatization and detection with an electrochemical detector $(\operatorname{method} B)$, were compared and the latter was found to be better. No significant change was observed within $24 \mathrm{~h}$ after irradiation in the levels of GSH and GSSG measured by method B. The GSH/GSSG ratio may be a less sensitive parameter for the evaluation of acute oxidative stress caused by X-ray irradiation in the skin. Monitoring the ascorbyl radical seems to be a good way to evaluate oxidative stress in skin in vivo.

Key words oxidative stress; mouse skin; ascorbyl radical; lipid peroxidation; glutathione

The skin is an organ functioning as a physical barrier to protect the body against hazards of the environment. Radiation is one such hazard and pathophysiological changes in the skin caused by X-ray irradiation include erythema, desquamation, and alopecia for early changes within hours and weeks and dermal atrophy and telangiectasia for later effects. $^{1-3)}$ The most serious manifestations are skin ulceration, necrosis and cancer.

Oxidative stress is considered one of the major causes of ionizing radiation-induced damage. ${ }^{4-7)}$ Exposure to ionizing radiation leads to the generation of extra reactive oxygen species and free radicals, ${ }^{8,9}$ which attack sensitive enzymes, constitutive proteins, DNA and membrane lipids. When the intrinsic repair and free radical scavenger systems are not able to counteract these insults, irreversible oxidative damage occurs.

To investigate the relationship between radiation-induced damage and oxidative stress, it is important to evaluate the oxidative stress quantitatively. However, appropriate markers for the evaluation of in vivo oxidative stress in the skin and a method for their measurement have not been fully established.

$\mathrm{X}$-Ray irradiation of aqueous solution produces free radicals such as the hydroxyl radical and hydrogen radical. ${ }^{9)}$ When lipids are attacked by such free radicals, a lipid peroxidation chain reaction occurs. Ascorbic acid and glutathione (GSH) are typical intrinsic antioxidants present in high concentrations in the body. Ascorbic acid reacts with free radicals at near diffusion-controlled rates to produce the ascorbyl radical and non-radical products. The ascorbyl radical is also produced by the recycling of vitamin $\mathrm{E}$ from its radical form by ascorbic acid. ${ }^{10)}$ Since the ascorbyl radical has a relatively long lifespan and is easily detectable even at room tempera- ture by ESR, the amount of ascorbyl radical may serve as a natural indicator of oxidative stress. ${ }^{11-13)}$ Glutathione (GSH) is a potent antioxidant and forms oxidized disulfide (GSSG) when exposed to oxidative stress. Since intracellular GSH is usually maintained in the reduced state, the GSH/GSSG ratio may be an indicator of oxidative stress. ${ }^{14,15)}$ In the present study, therefore, we examined lipid peroxidation, ascorbyl radical formation, and glutathione redox status as a possible quantitative marker for oxidative stress in mouse skin incurred by X-ray irradiation.

\section{Experimental}

Reagents Leupeptin, aprotinin, pepstatin, sodium orthovanadate, sodium fluoride, NADPH, bovine serum albumin, glutathione reductase, GSH and GSSG were products of Sigma (St. Louis, MO, U.S.A.). Metaphosphoric acid, fluorodinitrobenzene, trifluoroacetic acid, and 1-octane sulfonic acid were products of Wako Ltd. (Osaka, Japan). Bathophenanthroline disulfonic acid was obtained from Dojindo Laboratories $(\mathrm{Ku}-$ mamoto, Japan). Water was double distilled and treated with an ultrapure water apparatus (Simpli Lab, Nihon Millipore K.K., Tokyo, Japan).

X-Ray Irradiation of Mice Four-week-old male hairless mice (Hos:HR-1) were purchased from Japan SLC Co. (Hamamatsu, Japan) and kept in the animal house of the National Institute of Radiological Sciences, with water and food freely available. Whole-body irradiation of mice was performed using an X-ray generator (Pantak HF-320S, Shimadzu, Kyoto, Japan) at a dose rate of $1.0 \mathrm{~Gy} / \mathrm{min}$. The research was conducted with the approval of the institution's animal use committee.

Preparation of Skin Samples Skin tissue was removed from the backs of the mice at different time points after irradiation, immediately frozen in liquid nitrogen, and then stored at $-80^{\circ} \mathrm{C}$. For measuring lipid peroxidation and levels of glutathione, the frozen skin samples were pulverized into a powder in liquid nitrogen using a freezer mill (6750 Freezer/Mill, SPEX Certiprep, NJ, U.S.A.). For ESR measurements, skin tissue $(55 \mathrm{mg}$, $4.5 \times 0.6 \mathrm{~cm}^{2}, 0.6 \mathrm{~mm}$ in thickness) was stripped from the backs of the mice.

Lipid Peroxidation Measurements Hairless mice were divided into three groups of six, one for a control and two for 50 Gy whole-body X-ray irradiation. The skin samples of the irradiated groups were collected at $30 \mathrm{~h}$ and $78 \mathrm{~h}$ and stored at $-80^{\circ} \mathrm{C}$ after being frozen in liquid nitrogen. Lipid 
peroxidation was evaluated as thiobarbituric acid (TBA) reactive substances (TBARS) according to Ohkawa ${ }^{16)}$ with minor modifications. Briefly, the skin tissue was pulverized into a powder using a freezer/mill and homogenized with 4 volumes of a $1.15 \% \mathrm{KCl}$ solution in an ice-water bath. The reaction mixture contained $0.1 \mathrm{ml}$ of skin homogenate, $0.2 \mathrm{ml}$ of $8.1 \%$ sodium dodecyl sulfate, $1.5 \mathrm{ml}$ of a $20 \%$ acetic acid solution and $1.5 \mathrm{ml}$ of a $0.8 \%$ aqueous solution of TBA. The mixture was made up to $4 \mathrm{ml}$ with water and heated at $95^{\circ} \mathrm{C}$ for $1 \mathrm{~h}$. After cooling, the mixture was added to $1.0 \mathrm{ml}$ of water and $5.0 \mathrm{ml}$ of a mixture of $n$-butanol and pyridine $(15: 1, \mathrm{v} / \mathrm{v})$ and then shaken vigorously. After centrifugation at $3500 \mathrm{rpm}$ for $20 \mathrm{~min}$, the absorbance of the organic layer (upper layer) was measured at $532 \mathrm{~nm}$. The TBARS level was expressed as the amount of malondialdehyde (MDA), which was produced by the hydrolysis of 1,1,3,3-tetraethoxypropane as a standard.

ESR Measurements Skin stripped from the back of the mouse was placed into a tissue cell and ESR spectra were measured with an X-band ESR spectrometer (LFR-30, JEOL, Tokyo, Japan) at the following settings: frequency $9.4 \mathrm{GHz}$, power $4.0 \mathrm{~mW}$, field center $334.0 \mathrm{mT}$, sweep width $\pm 5.0 \mathrm{mT}$, sweep time $15 \mathrm{~min}$, modulation width $0.1 \mathrm{mT}$, gain 1000 , time constant $1.0 \mathrm{~s}$. The signal intensity is calculated as the ratio of signal height to a manganese marker placed in a cavity of the instrument. Simulation of the ESR spectrum was done using software commercially available (Radical Research Co., Tokyo, Japan).

Glutathione Measurements by HPLC after Derivatization (Method A) Skin tissue powder was mixed with 9 volumes of a buffer containing $6 \%$ metaphosphoric acid and $1 \mathrm{~mm}$ bathophenanthroline disulfonic acid, and homogenized in an ice-water bath. The suspension was centrifuged at $7790 \times \mathbf{g}$ for $5 \mathrm{~min}$ at $4{ }^{\circ} \mathrm{C}$ and the $\mathrm{pH}$ of the supernatant $(500 \mu \mathrm{l})$ was adjusted to 8.5 using $480 \mu \mathrm{l}$ of $2.4 \mathrm{M} \mathrm{KHCO}_{3}$ and about $120 \mu \mathrm{l}$ of $2 \mathrm{M} \mathrm{KOH}$. Derivatization was achieved by adding $1 \mathrm{ml}$ of 1\% 1-fluoro-2,4-dinitrobenzene (DNFB) in ethanol and incubating in the dark at $40{ }^{\circ} \mathrm{C}$ for $2 \mathrm{~h}$. Next, $150 \mu \mathrm{l}$ of $70 \%$ perchloric acid was added to the yellow reaction mixture and the mixture was centrifuged at $5600 \times \boldsymbol{g}$ for $5 \mathrm{~min}$. The supernatant was filtered through a $0.22 \mu \mathrm{m}$ polyvinylidene fluoride (PVDF) membrane filter ( $4 \mathrm{~mm}$ Millex GV, Millipore) and $15 \mu \mathrm{l}$ of the filtrate was applied to an HPLC system (Nanospace SI-2, Shiseido, Tokyo, Japan) with a UV detector (at $365 \mathrm{~nm}$ ). The HPLC system is of gradient capability and equipped with an auto-sampler. Mobile phase A was $0.1 \%$ trifluoroacetic acid in water and mobile phase B was $0.1 \%$ trifluoroacetic acid in acetonitrile. The column was a $\mathrm{C} 18, \mathrm{MG}, 5 \mu \mathrm{m}, 2.0 \mathrm{~mm} \phi \times 250 \mathrm{~mm}$ (Shiseido, Japan). The analysis time for one sample was $55 \mathrm{~min}$.

Glutathione Measurements by HPLC with the Electrochemical Detector (Method B) With the same procedure as method A, skin tissue powder was mixed with 9 volumes of a buffer containing $6 \%$ metaphosphoric acid and $1 \mathrm{~mm}$ bathophenanthroline disulfonic acid, and homogenized in an icewater bath. The supernatant was filtered through a $0.22 \mu \mathrm{m}$ PVDF membrane filter ( $4 \mathrm{~mm}$ Millex GV, Millipore) and the filtrate was directly applied to an HPLC system with a coulometric electrochemical detector. The HPLC system consists of a high pressure pump (Model 582, ESA, Inc., Bedford, MA, U.S.A.), a hand injector (Rheodyne 7161) with a $100 \mu$ linjection loop, and a C18 column $(4.6 \mathrm{~mm} \phi \times 250 \mathrm{~mm}$, MC Medical Co., Tokyo, Japan). The elution was $0.6 \mathrm{ml} / \mathrm{min}$ and the mobile phase contained $0.1 \mathrm{M}$ phosphate buffer ( $\mathrm{pH} 2.6$ adjusted with $85 \%$ phosphoric acid), $0.15 \mathrm{~mm}$ 1-octane sulfonic acid, $4 \%$ methanol, and $0.005 \% \mathrm{KCG}$ reagent (ESA, Inc.). The detector was a Coulochem II (ESA, Inc.) with a Model 5010 analytical cell containing two porous graphite electrodes and a Model 5020 guard cell. The guard cell was set at $+900 \mathrm{mV}$, electrode 1 at $+700 \mathrm{mV}$, and electrode 2 at $+850 \mathrm{mV}$. Full-scale output was set at $50 \mu \mathrm{A}$ for electrode 1 and $1 \mu \mathrm{A}$ for electrode 2. Recording and analysis of the chromatogram was performed using commercial software (CHROMELEON ${ }^{\mathrm{TM}}$ chromatography managing system, Dionex Co., Sunnyvale, CA, U.S.A.).

Measurement of Glutathione Reductase Activity The glutathione reductase activity was measured using an enzymatic coupling of the reduction of GSSG to GSH with the oxidation of reduced nicotinamide adenine dinucleotide phosphate (NADPH) to nicotinamide adenine dinucleotide phosphate (NADP). A solution containing $200 \mu 1$ of $100 \mathrm{~mm}$ ethylenediaminetetraacetic acid buffer ( $\mathrm{pH} 7.6), 50 \mu \mathrm{l}$ of $1 \mathrm{~mm} \mathrm{NADPH}, 50 \mu \mathrm{l}$ of $10 \mathrm{~mm}$ GSSG, $50 \mu \mathrm{l}$ of $10 \mathrm{mg} / \mathrm{ml}$ bovine serum albumin, and $100 \mu \mathrm{l}$ of water was pre-incubated at $25^{\circ} \mathrm{C}$ for $5 \mathrm{~min}$. The solution was transferred to a cuvette and $50 \mu \mathrm{l}$ of a sample solution or standard glutathione reductase solution was added, and then absorbance at $340 \mathrm{~nm}$ was continuously measured at $25^{\circ} \mathrm{C}$.

Statistical Analysis An analysis of variance (ANOVA) was used to compare the difference between groups. The difference between two groups

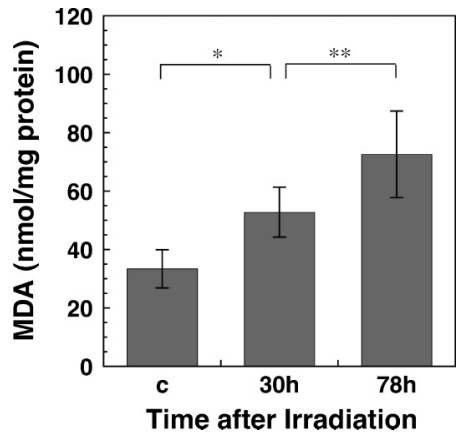

Fig. 1. Lipid Peroxidation of Mouse Skin after X-Ray Irradiation

We used two irradiated and one sham-control group of six mice each. Skin samples were collected without irradiation and at $30 \mathrm{~h}$ and $78 \mathrm{~h}$ after irradiation. The skin homogenate was used to measure malondialdehyde levels with a thiobarbituric acid assay. The values are means \pm S.E. obtained for 6 mice each. Statistical significance: $* p<0.05, * * p<0.01$.

was evaluated with the Newman-Keuls test. The level of significance was taken as $p<0.05$.

\section{Results}

Lipid Peroxidation of the Mouse Skin after X-Ray Irradiation Figure 1 shows the change in the lipid peroxidation level of mouse skin homogenate after 50 Gy of X-ray irradiation. The lipid peroxidation measured as the MDA level progressed with time after the irradiation. The level was significantly higher than the control at $30 \mathrm{~h}$ after the irradiation and about twice the control value at $78 \mathrm{~h}$.

Effects of X-Ray Irradiation on the Ascorbyl Radical in the Skin Figure 2A shows ESR spectra of the ascorbyl radical in mouse skin. A weak doublet signal was observed in spectrum a), which was obtained for the skin sample before irradiation. The signals at both ends are the third and fourth lines of the manganese signal used for a marker. A stronger doublet signal was observed for the skin sample obtained at $78 \mathrm{~h}$ after X-ray irradiation b). The doublet signal was simulated with a computer and the simulated spectrum is shown in c). The parameters, hyperfine splitting of $0.1833 \mathrm{mT}$ and a $g$-value of 2.0052 , correspond to the ascorbyl radical. ${ }^{12,17)}$ Figure $2 \mathrm{~B}$ shows the time course of signal intensity of the ascorbyl radical after X-ray irradiation. The signal gradually strengthened with time after the irradiation. Similar to the lipid peroxidation level shown in Fig. 1, the signal intensity was significantly greater at $30 \mathrm{~h}$ and about twice the control value at $78 \mathrm{~h}$ post-irradiation (Fig. 2C). Since we used different manganese markers for the experiments in Figs. 2B and $\mathrm{C}$, we cannot directly compare the signal intensity of those figures shown as relative intensity to the manganese marker.

Effects of X-Ray Irradiation on Skin Glutathione Concentration At first we assessed two HPLC methods (method A and method B) of measuring glutathione (GSH) and its oxidized form (GSSG) simultaneously. Method A derivatizes GSH and GSSG with DNFB and detects them with a UV-visible detector after separation with an HPLCcolumn. Method B separates GSH and GSSG on an HPLC column without derivatization and detects them with an electrochemical detector. When a tissue sample was used to measure GSH and GSSG, both methods showed acceptable linearity and recovery (data not shown). The sensitivity of the two methods was similar. When commercial GSH and GSSG 
(A)

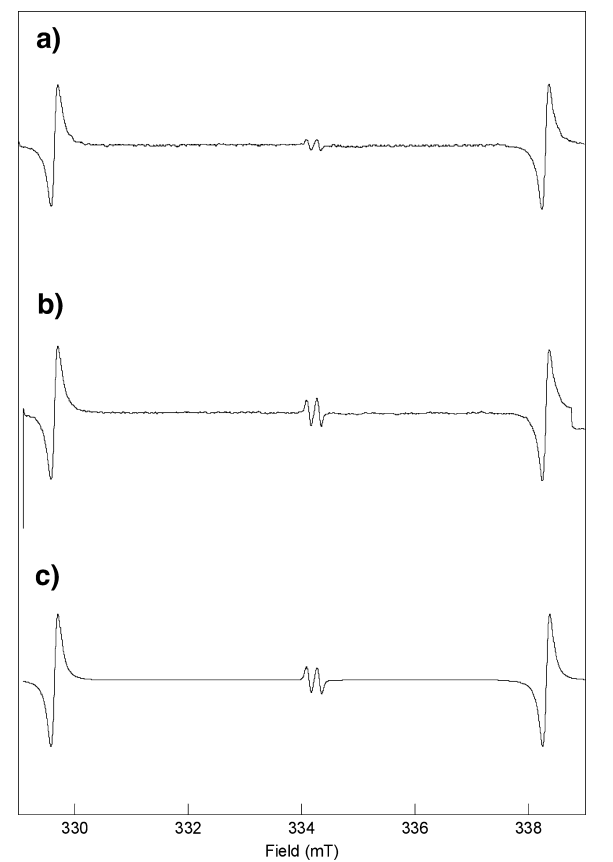

(B)

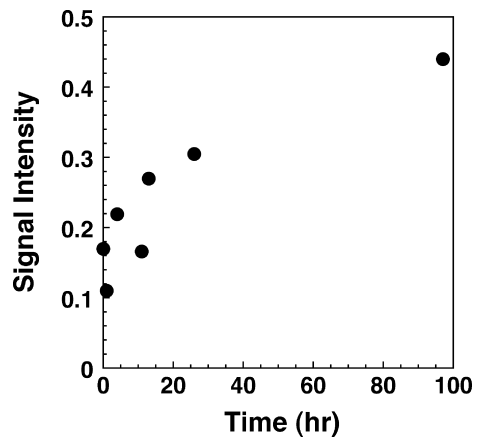

(C)

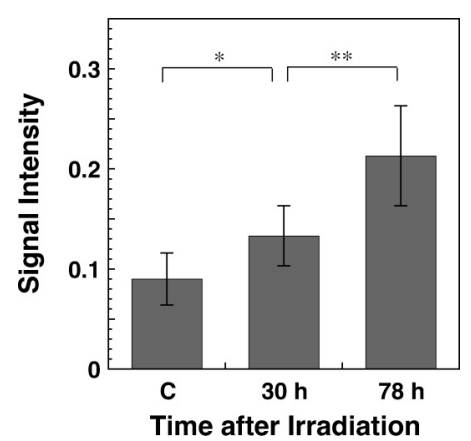

Fig. 2. Change in Amount of the Ascorbyl Radical in Mouse Skin after X-Ray Irradiation

(A) a) An ESR spectrum of a skin sample prepared from a mouse without irradiation. b) An ESR spectrum of a skin sample prepared from a mouse at $78 \mathrm{~h}$ after $50 \mathrm{~Gy}$ of X-ray irradiation. c) A simulated spectrum using parameters of $0.1833 \mathrm{mT}$ for hyperfine splitting and 2.0052 for the $g$-value. The doublet signal at the center corresponds to the ascorbyl radical. The strong signals at both ends are from standard manganese. (B) Time-course of the change in the amount of ascorbyl radical in mouse skin after $50 \mathrm{~Gy}$ of X-ray irradiation. At each time point after the irradiation, a strip of skin $(55 \mathrm{mg}$, area $=4.5 \times 0.6 \mathrm{~cm}$, thickness $=0.6 \mathrm{~mm})$, was cut from the mouse and put into a tissue cell, and then X-band ESR spectra were measured to quantify the amount of ascorbyl radical in the skin. (C) Signal intensity of ascorbyl radical in mouse skin after 50 Gy of X-ray irradiation. We used two irradiated and one sham-control group of six mice each. Skin samples were collected without irradiation and at $30 \mathrm{~h}$ and $78 \mathrm{~h}$ after irradiation. The signal intensity is calculated as the ratio of signal height to a manganese marker. The values are means \pm S.E. obtained for 6 mice each. Statistical significance: $* p<0.05, * * p<0.01$.

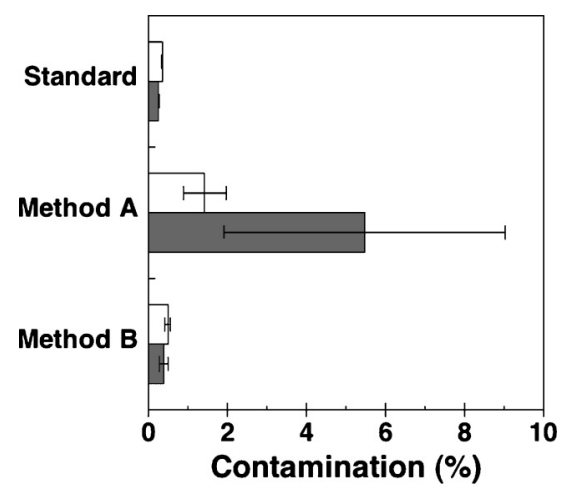

Fig. 3. Comparison of Two HPLC Methods for Simultaneous Measurement of GSH and GSSG

Standard GSH and GSSG solutions (100 mm each) were prepared from commercial GSH and GSSG and the purity of each standard solution was evaluated by HPLC with a UV detector $(215 \mathrm{~nm})$. The proportion of GSSG in the standard GSH solution was $0.35 \%$ (open bar of Standard). That of GSH in the standard GSSG solution was $0.26 \%$ (hatched bar of Standard). The standard GSH and GSSG solutions were diluted to $1 \mathrm{~mm}$ and the contents of GSSG in GSH (open bar) and GSH in GSSG (hatched bar) were measured with methods A and B. For a description of methods A and B, see the Materials and Methods section in the text. The values are means \pm S.D. $(n=3)$.

were measured, method B provided less impurity (GSSG in GSH, and GSH in GSSG) than method A and the value was almost equal to the expected one (Fig. 3). In addition, method B needed less handling to prepare the sample than did method A. Therefore, we used method B for later measurements of GSH and GSSG.

Table 1 shows the amount of GSH and GSSG in different tissues of mice. As shown in the table, skin has more GSSG
Table 1. GSH and GSSG Concentrations in Several Tissues of Mice

\begin{tabular}{lccc}
\hline \hline & Skin & Liver & Brain \\
\hline GSH (nmol/g tissue) & $337 \pm 68$ & $3185 \pm 355$ & $1627 \pm 86$ \\
GSSG (nmol/g tissue) & $39.3 \pm 13.9$ & $15.2 \pm 9.4$ & $4.4 \pm 2.6$ \\
GSH/GSSG & 8.6 & 210 & 370 \\
\hline
\end{tabular}

Concentrations of GSH and GSSG in tissue homogenates were measured by method $\mathrm{B}$ as described in the text. The values are means \pm S.D. $(n=4$ for skin and 3 for liver and brain).

and less GSH than the liver and brain.

Figures 4A and B show the amount of GSH and GSSG, respectively, in mouse skin irradiated with 50 Gy X-rays. No significant change was observed for GSH or GSSG even at $24 \mathrm{~h}$ after the irradiation. In a different experiment where a $1 \mathrm{ml}$ of a $200 \mu \mathrm{M}$ GSH aqueous solution in a test tube was irradiated with $50 \mathrm{~Gy}$, the GSH concentration decreased to $195 \mu \mathrm{M}$, whereas $2.3 \mu \mathrm{M}$ of GSSG appeared. The activity of glutathione reductase increased at $2 \mathrm{~h}$ but recovered at $24 \mathrm{~h}$ after the irradiation (Fig. 4C).

\section{Discussion}

In general, X-ray irradiation of biological samples produces free radicals, which cause lipid peroxidation. Previously, we have reported that lipid peroxidation occurs in mouse liver after 15 Gy of whole body X-ray irradiation. ${ }^{18)} \mathrm{In}$ the present study, we show that lipid peroxidation also occurs in mouse skin after X-ray irradiation (Fig. 1A).

We also found that the amount of the ascorbyl radical in the skin increases after X-ray irradiation. Since this increase 
(A)

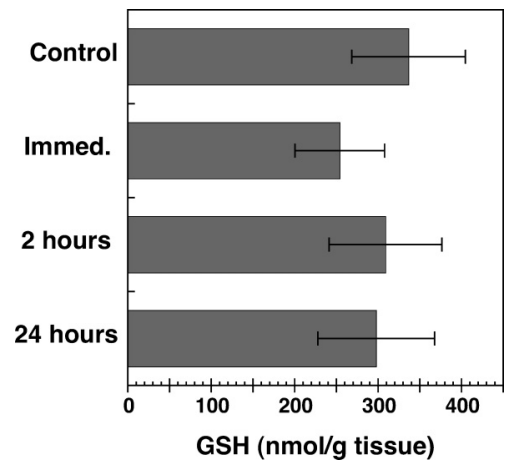

(B)

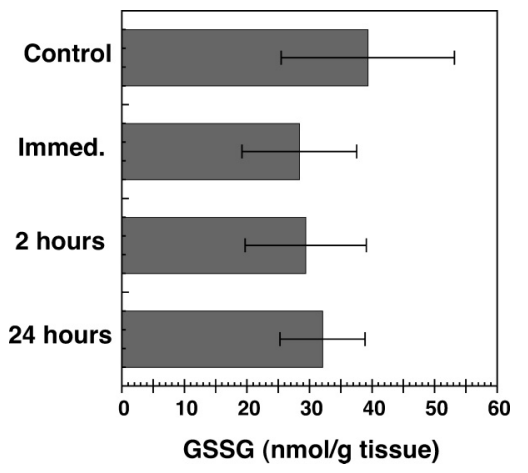

(C)

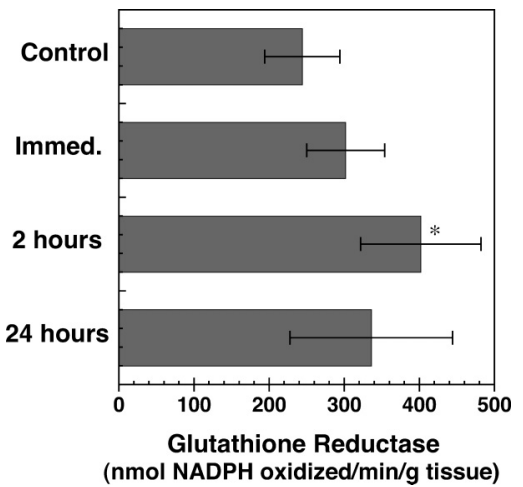

Fig. 4. Change in Levels of GSH (A), GSSG (B), and Glutathione Reductase Activity (C) in X-Ray Irradiated Mouse Skin

Content and activity were measured in skin homogenate prepared from mice without irradiation (Control), immediately after irradiation (Immed.), $2 \mathrm{~h}$ after irradiation $(2 \mathrm{~h})$ and $24 \mathrm{~h}$ after irradiation $(24 \mathrm{~h})$. Statistical significance: $* p<0.05$.

parallels the increase in lipid peroxidation (increase of TBARS), the change may reflect oxidative stress in the skin. Lipid peroxidation is prevented by vitamin $\mathrm{E}$; the vitamin $\mathrm{E}$ radical formed in the membrane phase is reduced back to vitamin $\mathrm{E}$ by ascorbic acid in the water phase forming ascorbyl radical. ${ }^{19-22)}$ This recycling reaction could be one of the mechanisms for producing ascorbyl radical in the skin. Finding the parallel increase of lipid peroxidation and ascorbyl radical after $\mathrm{X}$-irradiation support this hypothesis. There are several reports that the production of the ascorbyl radical is related to oxidative stress in biological systems. Timmins and Davis reported that measuring the amount of ascorbyl radical by ESR spectroscopy is useful for studying the production of free radicals in intact tissues. ${ }^{23}$ ) It was reported that the amount of ascorbyl radical in plasma increased significantly in rats under oxidative stress caused by iron overloading. ${ }^{12)}$ The level of ascorbyl radical in cerebrospinal fluid reportedly had a strong correlation in acute lymphoblastic leukemia patients undergoing chemotherapy. ${ }^{17)}$ Since attempts to spin trap radicals in skin irradiated with UV or ionizing radiation have been unsuccessful and ascorbic acid is a natural intrinsic antioxidant present at high concentrations in tissues, the ascorbyl radical may be a good marker for the quantitative evaluation of oxidative stress in the living body as indicated in this study.

Glutathione (GSH) is also an intrinsic antioxidant whose concentration in tissues is high. When oxidized, a dimer (GSSG) is formed and the equilibrium between GSH and GSSG is thought to be a possible marker reflecting the redox status of a living body. ${ }^{15,24)}$ In fact, Navarro et al. have reported that X-ray irradiation increases blood GSSG. ${ }^{25)}$ In the present study, we examine whether the redox equilibrium of GSH and GSSG can be used as a marker for oxidative stress in mouse skin. For this purpose, it is important to measure the amounts of GSH and GSSG simultaneously. HPLC is best for the quantitative measurement of GSH and GSSG and two reported methods seemed to be good: detection with a UV-visible detector after sample derivatization with 1-fluoro-2,4-dinitrobenzene $(\mathrm{DNFB})(\operatorname{method} \mathrm{A})^{26-28)}$ and direct detection with an electrochemical detector (method B). ${ }^{29-32)}$ First, we examined which method best suits our purposes and found that method B is superior because: 1) There was less conversion to the other component (GSH to GSSG or GSSG to GSH) with method B. Method A has a process of derivatization reaction, during which conversion of GSH to GSSG and GSSG to GSH could occur. When old DNFB was used for derivatization, more conversion (artifact) was observed with method A (data not shown). 2) Less handling is required for sample preparation using method B. 3) Although the sensitivity was similar between the two methods, less starting sample volume was required for method B than method A.

When mice were irradiated with a high dose $(50 \mathrm{~Gy})$ of $\mathrm{X}$-rays, no significant change in the concentrations of GSH and GSSG was observed. When the GSH solution was irradiated with $50 \mathrm{~Gy}$, a reduction in the amount of GSH and the production of GSSG were observed. A yield of $2.3 \mu \mathrm{M}$ of GSSG could be produced by the reaction with $4.6 \mu \mathrm{M}$ of the hydroxyl radical, which corresponds to about $30 \%$ of the radical calculated to be produced by $50 \mathrm{~Gy}$ irradiation of water based on the $G$ value (2.7) of hydroxyl radical formation by radiation. Glutathione couples various metabolic reactions in living systems. Therefore, the absence of a significant change in the levels of GSH and GSSG suggests strong homeostatic metabolism, which may recover GSH by reducing the oxidative GSSG. The increase in glutathione reductase activity observed at $2 \mathrm{~h}$ after irradiation may imply such a metabolic response. In addition, there is a GSSG export system in erythrocytes and liver cells. ${ }^{33)}$ If such a system exists in skin cells, the export of GSSG from the skin cells might also contribute to the absence of significant change in the GSH/ GSSG ratio.

It is interesting that the skin has a higher concentration of GSSG and lower GSH/GSSG value than the liver or brain. Since the skin is exposed to various forms of environmental stress, it is reasonable for oxidative stress to be greater in the skin than in the inner organs.

In conclusion, among the parameters examined, that is, the level of lipid peroxidation, the amount of ascorbyl radical, and the GSH/GSSG ratio, the amount of ascorbyl radical 
may be best for the quantitative evaluation of oxidative stress in the skin, because it increases with time after X-ray irradiation and measuring it is easier than measuring lipid peroxidation. In the present study, we used stripped skin samples for ESR measurements, which restrict the application of this method. If the sensitivity of in vivo ESR instruments can be improved, one could measure the amount of ascorbyl radical in the skin of living mice and a non-invasive evaluation of oxidative stress in the skin could be possible.

Acknowledgments We thank Mr. Daisuke Hayashi for help with simulation of ESR spectrum. This work was supported in part by grants from the Sasakawa Scientific Research Grant of the Japan Science Society and from the Nakatomi Foundation.

\section{References}

1) Kiefer J., "Biological Radiation Effects," Springer-Verlag, Berlin, 1989, pp. $291-308$.

2) Grosch D. S., Hopwood L. E., "Biological Effects of Radiations," Academic Press, New York, 1979, pp. 192-208.

3) Hopewell J. W., Int. J. Radiat. Biol., 57, 751-773 (1990).

4) Blattner C., Herrlich P., "Handbook of Cell Signaling," Vol. 2, ed. by Bradshaw R. A., Dennis E. A., Academic Press, New York, 2004, pp. $257-262$.

5) Demple B., "Handbook of Cell Signaling," Vol. 2, ed. by Bradshaw R. A., Dennis E. A., Academic Press, New York, 2004, pp. 191-195.

6) Mcbride H. W., Iwamoto S. K., Syljuasen R., Pervan M., Pajonk F., Oncogene, 22, 5755-5773 (2003).

7) Criswell T., Leskov K., Miyamoto S., Luo G. B., Boothman D. A., Oncogene, 22, 5813-5827 (2003).

8) Mitchell J. B., Russo A., Kuppusamy P., Krishna M. C., Ann. N.Y. Acad. Sci., 899, 28-43 (2000).

9) Moritake T., Tsuboi K., Anzai K., Ozawa T., Ando K., Nose T., Radiat. Res., 159, 670-675 (2003).

10) Packer J. E., Slater T. F., Willson R. L., Nature (London), 278, 737738 (1979).

11) Roginsky V. A., Stegmann H. B., Free Radic. Biol. Med., 17, 93-103 (1994).
12) Galleano M., Aimo L., Puntarulo S., Toxicol. Lett., 133, 193-201 (2002).

13) Buettner G. R., Jurkiewicz B. A., Free Radic. Biol. Med., 14, 49-55 (1993).

14) Asensi M., Sastre J., Pallardo F. V., Lloret A., Lehner M., Garcia-DeLa Asuncion J., Vina J., Methods Enzymol., 299, 267-276 (1999).

15) Schafer F. Q., Buettner G. R., Free Radic. Biol. Med., 30, 1191-1212 (2001).

16) Ohkawa H., Ohishi N., Yagi K., Anal. Biochem., 95, 351-358 (1979).

17) Nakagawa K., Kanno H., Miura Y., Anal. Biochem., 254, 31-35 (1997).

18) Miura Y., Anzai K., Urano S., Ozawa T., Free Radic. Biol. Med., 23, 533-540 (1997).

19) May J. M., Qu Z. C., Mendiratta S., Arch. Biochem. Biophys., 349, $281-289$ (1998).

20) Waters R. E., 2nd, White L. L., May J. M., Free Radic. Res., 26, $373-$ 379 (1997).

21) Constantinescu A., Han D., Packer L., J. Biol. Chem., 268, 10906 10913 (1993).

22) Halpner A. D., Handelman G. J., Harris J. M., Belmont C. A., Blumberg J. B., Arch. Biochem. Biophys., 359, 305-309 (1998).

23) Timmins G. S., Davies M. J., Carcinogenesis, 14, 1499-1503 (1993).

24) Jones D. P., Methods Enzymol., 348, 93-112 (2002).

25) Navarro J., Obrador E., Pellicer J. A., Aseni M., Vina J., Estrela J. M., Free Radic. Biol. Med., 22, 1203-1209 (1997).

26) Dominick P. K., Cassidy P. B., Roberts J. C., J. Chromatogr. B., 761, $1-12$ (2001).

27) Giustarini D., Dalle-Donne I., Colombo R., Milzani A., Rossi R., Free Radic. Biol. Med., 35, 1365-1372 (2003).

28) Yoshida T., J. Chromatogr. B., 678, 157-164 (1996).

29) Lakritz J., Plopper C. G., Buckpitt A. R., Anal. Biochem., 247, 63-68 (1997).

30) Rodriguez-Ariza A., Toribio F., Lopez-Barea J., J. Chromatogr. B., 656, $311-318$ (1994).

31) Smith N. C., Dunnett M., Mills P. C., J. Chromatogr. B., 673, 35-41 (1995).

32) Remiao F., Carmo H., Carvalho F., Bastos M. L., Biomed. Chromatogr., 14, 468-473 (2000).

33) Halliwell B., Gutteridge J. M. C., "Free Radicals in Biology and Medicine," 3rd ed., Oxford University Press Inc., London, 1999. 